\title{
ESTUDO EXPERIMENTAL E DE SIMULAÇÃO CFD DO GOTEJAMENTO DE ÓLEO A PARTIR DE UMA PEQUENA FONTE CILÍNDRICA SUBMERSA EM ÁGUA
}

\author{
D. R. SIMIONI ${ }^{1}$, M. B. QUADRI ${ }^{1}$, A. BOLZAN ${ }^{1}$ \\ ${ }^{1}$ Universidade Federal de Santa Catarina, Departamento de Engenharia Química \\ e Engenharia de Alimentos. \\ E-mail para contato: m-quadri@enq.ufsc.br, debora.simioni@hotmail.com.br
}

\begin{abstract}
RESUMO - A exploração de petróleo offshore em profundidade está sujeita a condições que afetam propriedades físico-químicas dos fluidos. Tanto em situações de operação como de vazamentos acidentais, o estudo destas propriedades e padrões de escoamento é importante. Com essa motivação, desenvolve-se um estudo do escoamento óleo/água a partir de experimentos e simulações CFD em geometria tridimensional por meio do software COMSOL Multiphysics 4.3b. Além das equações de Navier-Stokes e equação da conservação da massa, emprega-se o método Level Set de acompanhamento da interface. Sendo a técnica numérica de elementos finitos. Os experimentos consistiram na injeção, por meio de um bocal cilíndrico, de óleo de soja, em água destilada à $22^{\circ} \mathrm{C}$. Os aspectos avaliados foram: altura de gotejamento do óleo, diâmetro médio das gotas e tempo entre liberação de gotas. As simulações contemplaram três velocidades de injeção do óleo e reproduziram adequadamente o comportamento experimental. $\mathrm{O}$ diâmetro médio das gotas simuladas apresentou desvio máximo de $\pm 1,3 \%$. Os resultados representaram adequadamente, tanto qualitativa quanto quantitativamente, o comportamento fluidodinâmico dos experimentos, atestando a pertinência da metodologia utilizada e estabelecendo este tipo de modelo como um excelente ponto de partida para a consideração de problemas de escoamento de óleo a partir de uma fonte submersa.
\end{abstract}

\section{INTRODUÇÃO}

O interesse pelo estudo da fluidodinâmica e de propriedades físicas de sistemas óleo/água se justifica pela ampla gama de aplicações na engenharia das operações offshore de petróleo. Situações de vazamento geradas por falhas em equipamentos e tubulações, além das características geológicas dos sítios de exploração que podem levar ao fenômeno da exsudação de óleo, são exemplos de eventos que exigem soluções efetivas de combate aos danos ambientais e redução de prejuízos na produção. Quando ocorre um vazamento em estágio de gotejamento, pode-se identificar uma sucessão de etapas até o óleo chegar à superfície da água: (1) a formação inicial da gota; (2) o crescimento da gota junto à borda do orifício; (3) a formação de um 
prolongamento (pescoço) na base da gota; e (4) o desprendimento da gota de sua fase de origem e ascensão através da fase contínua, (TIMGREN et.al., 2007). Este tipo de fenômeno tem sido modelado através da dinâmica de fluidos computacional (CFD) por diferentes pesquisadores, tais como Li, et.al.,(2012) e Kobayashi, ( 2007). As principais forças que atuam sobre uma gota antes da sua separação estão esquematicamente representadas na Figura 1. As forças são descritas

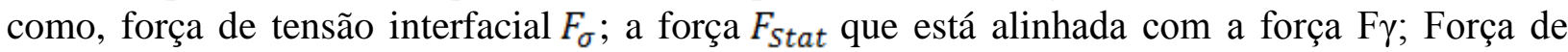
Empuxo $F_{E}$; força de sustentação dinâmica $F_{D}$; força de arrasto do viscoso $F_{R}$; força de momento linear $F_{1}$, (ZHI et.al., 1999). Dentre essas forças, $F_{\sigma}$ é uma força de retenção, enquanto $F_{S t a t}, F_{E}$, $F_{D}, F_{R}$ e $F_{1}$ são forças que promovem o destacamento da gota. $\mathrm{O}$ aumento em $F_{\sigma}-F_{S t a t}$ irá aumentar o diâmetro das gotas no momento do desprendimento, ao passo que o aumento da $F_{E}$, $F_{D}, F_{R}$ e $F_{1}$ irá diminuir o diâmetro das gotas.

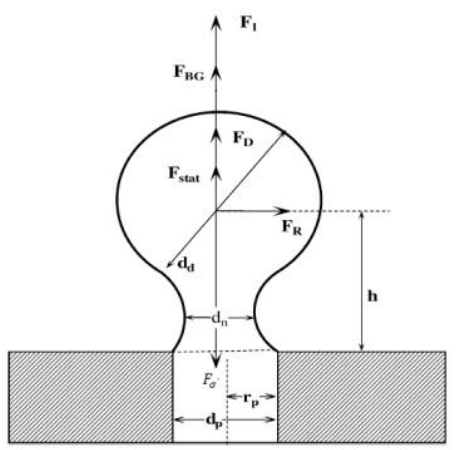

Figura 1-Diagrama esquemático das forças atuantes na gota. Fonte: Adaptado de XU et. al.,2005.

Assim, este trabalho tem por objetivo desenvolver um estudo teórico/experimental do vazamento por gotejamento de óleo em meio subaquático no que tange aos aspectos de formação, desprendimento e ascensão da gota em direção à superfície. Busca-se representar de forma realística essa sucessão de fenômenos físicos por meio da fluidodinâmica computacional (CFD), a fim de contribuir com os esforços de modelagem e simulação aplicados às situações reais de vazamentos subaquáticos.

\section{MATERIAL E MÉTODOS}

\subsection{Etapa Experimental}

Neste subitem são apresentadas as avaliações das propriedades físico-químicas dos fluidos que apresentam significativa importância na formação da gota, diâmetro médio, altura de gotejamento e nos aspectos fenomenológicos do escoamento bifásico, que fazem parte deste estudo. Também é apresentado o aparato e os procedimentos experimentais seguidos.

Determinação da Tensão Interfacial: utilizou-se o método da gota pendente invertida, realizada no goniômetro Ramé-Hart, modelo-250-F1. As análises foram realizadas em triplicata a $22^{\circ} \mathrm{C}$. O resultado foi obtido pela média dos valores após a estabilização da tensão interfacial. 


\section{9 a 22 de outubro de 2014 \\ Florianópolis/SC}

Ângulo de contato: utilizou-se o mesmo equipamento utilizado para tensão interfacial. As análises foram realizadas em duplicata a $22^{\circ} \mathrm{C}$. O resultado foi obtido pela média dos ângulos de contato para gotas com cerca de $25 \mathrm{~mm}^{3}$.

Determinação da Viscosidade: As análises de viscosidade foram realizadas em um viscosímetro Brookfield Digital Modelo DV-III, utilizando geometria para baixas viscosidades. Os ensaios foram realizados em triplicata a temperatura de $22^{\circ} \mathrm{C}$.

Determinação da Densidade: A avaliação da densidade do óleo foi realizada a temperatura de $22^{\circ} \mathrm{C}$, com densímetro flutuante.

Aparato e procedimentos experimentais: utilizou-se um recipiente cilíndrico encamisado, com diâmetro interno de $0,04 \mathrm{~m}$ e altura de $0,4 \mathrm{~m}$. Este recipiente tem uma abertura na parte inferior com rolha de silicone transpassada por uma agulha de 0,0006 m de diâmetro interno; na parte superior o recipiente é aberto. Com a coluna preenchida com água destilada, o óleo é injetado através da agulha que é conectada a uma seringa através de uma mangueira. As velocidades de injeção de óleo testadas foram: $0,225 \mathrm{~m} / \mathrm{s}, 0,333 \mathrm{~m} / \mathrm{s}$ e $0,446 \mathrm{~m} / \mathrm{s}$. Os diâmetros médios experimentais das gotas formadas no sistema de injeção, bem como as alturas de gotejamento, foram calculados com base em fotografias obtidas durante os experimentos. Os diâmetros e alturas de gotejamento obtidos nas simulações foram quantificados a partir das imagens construídas com o software de simulação utilizado.

\subsection{Etapa de Modelagem}

O Método dos Elementos Finitos foi utilizado para a discretização e resolução das equações do escoamento e de acompanhamento da interface, empregando-se para isso a plataforma fornecida pelo software COMSOL Multiphysics 4.3b. As equações da continuidade e de Navier-Stokes para fluidos incompressíveis, incluindo o tensor interfacial, descrevem o escoamento do sistema bifásico. Os dois líquidos envolvidos neste problema podem ser considerados incompressíveis. Para este caso, a equação de Navier-Stokes é dada por:

$\rho \frac{\partial u}{\partial t}+\rho(u \cdot \nabla) u=\nabla \cdot\left[-\rho \mathbf{I}+\mu\left(\nabla u+\nabla u^{T}\right)\right]+F_{g}+F_{\sigma}+F_{E}+F_{A}$

Onde: $\rho$ é a densidade $\left(\mathrm{kg} / \mathrm{m}^{3}\right), \mu$ é a viscosidade dinâmica (Pa.s), $u$ é a velocidade $(\mathrm{m} / \mathrm{s}), p$ é a pressão $(\mathrm{Pa}), F_{g}$ é a força da aceleração gravitacional, $F_{\sigma}$ é a força da tensão interfacial, $F_{E}$, é a força de empuxo e $F_{A}$, é a força de arraste na interface, tendo envolvida em sua formulação a função delta Dirac $(\delta)$ que só não é nula na interface. Na formulação diferencial das equações de Navier-Stokes, a localização das forças de tensão superficial singulares, são representadas por uma função delta de Dirac, (TORNBERG, ENGQUIST, 2000). Para problemas de escoamento multifásicos é importante a localização e acompanhamento da interface, sendo que para isso foi utilizado o método Laminar Two-Phase Flow Level Set (OLSSON e KREISS, 2005). Este método utiliza um reinicializador $\gamma$, para preservar a equação do método Level Set, que descreve a convecção na interface dos fluidos. O contorno de 0,5 da função Level Set $(\phi)$ define a 


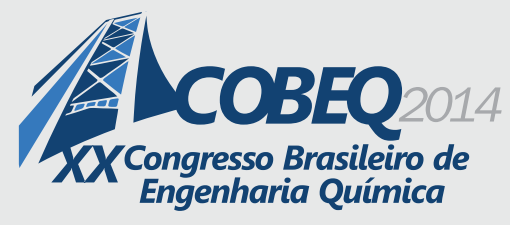

19 a 22 de outubro de 2014
Florianópolis/SC

interface entre os fluidos. Em uma camada de transição próxima à interface, $\phi$ é suavizado de 0 até 1 . A interface se move com o fluído a mesma velocidade, $u$. A equação que descreve a convecção de reinicialização da Função Level Set é dada por:

$\frac{\partial \phi}{\partial t}+u \cdot \nabla \Phi=\gamma \nabla \cdot\left(\varepsilon \nabla \Phi-\Phi(1-\Phi) \frac{\nabla \phi}{|\nabla \phi|}\right)$

O parâmetro $\gamma$ determina o incremento da reinicialização, sendo que seu valor máximo adequado deve ser da mesma magnitude da máxima velocidade atingida pelo sistema. Paralelamente à definição da interface, a função Level Set usa um suavizador de densidade e viscosidade que é aplicado na transição através da interface.

Geometria: o domínio de simulação é constituído de um cilindro de $0,03 \mathrm{~m}$ de altura e 0,012 m de diâmetro. Na parte superior do domínio tem-se uma camada de 0,0025m de óleo e centralizado na base do domínio posicionou-se outro pequeno cilindro (agulha) de 0,0006m de diâmetro interno e altura de $0,0025 \mathrm{~m}$. Foi adicionado um work plane na altura de saída do bocal, com geometrias circulares distribuídas, para refinar a malha na região onde ocorre o escoamento das gotas.

Condições Iniciais e de Contorno: Inicialmente, o óleo de soja ocupa os subdomínios no interior da agulha e na camada superior de óleo livre; o restante é ocupado pela água. Um perfil hidrostático foi aplicado a todo o domínio. Na base da agulha prescreve-se a entrada com velocidade de injeção especificada; já a saída, no limite superior do domínio, tem prescrita a condição de pressão zero. Para as paredes internas e borda do bocal da agulha adotou-se a condição de parede molhada, com ângulo de contato $\theta$ especificado. As demais paredes foram consideradas sem escorregamento $(u=0)$.

Malha: As malhas utilizadas nas simulações foram construídas de forma estruturada com elementos hexaédricos, totalizando 204.544 elementos. Essa escolha é justificada pelo estudo de malha realizado previamente.

Simulações: As simulações foram realizadas com o emprego do software COMSOL Multiphysics $4.3 b^{\circledR}$, em um computador com processador Core i7, 4700MQ, com 16GB de RAM e placa de vídeo dedicada com $2 \mathrm{~Gb}$. Após identificar os parâmetros de maior influência, estes foram ajustados e utilizaram-se os que apresentaram melhores resultados de diâmetro médio de gota formada para cada velocidade de injeção. Para todas as velocidades: Parâmetro de reinicialização $\gamma=0,05 \mathrm{~m} / \mathrm{s}$; Ângulo de contato $\theta=73^{\circ}$. Para as velocidades de 0,225 e $0,446 \mathrm{~m} / \mathrm{s}$ o parâmetro de Tensão interfacial foi de $\sigma=0,036 \mathrm{~N} / \mathrm{m}$ e para velocidade de $0,333 \mathrm{~m} / \mathrm{s}$ o parâmetro de Tensão interfacial foi de $\sigma=0,038 \mathrm{~N} / \mathrm{m}$. O ângulo de contato exigiu um estudo próprio e mais detalhado, assim além dos dados experimentais foi realizado um estudo com as simulações para comprovar a sensibilidade do simulador quanto a este parâmetro. Utilizou-se para estas simulações as propriedades físico-químicas avaliadas experimentalmente, velocidade de injeção de $0,0413 \mathrm{~m} / \mathrm{s}$, parâmetro de reinicialização gamma $\gamma=0,05 \mathrm{~m} / \mathrm{s}$, tensão interfacial $\sigma=$ $0,03 \mathrm{~N} / \mathrm{m}$, fazendo-se variar somente o ângulo de contato $\theta$. Inicialmente avaliou-se de $65^{\circ}$ a $85^{\circ}$, 
com intervalo de 5 em $5^{\circ}$, sendo apresentado um valor de máximo entre $70^{\circ}$ e $75^{\circ}$, assim este intervalo foi explorado de $1 \mathrm{em} 1^{\circ}$. Os resultados percentuais comparativos para o diâmetro médio de gota simulado frente ao experimental são apresentados na Tabela 1.

\section{RESULTADOS E DISCUSSÕES}

\subsection{Propriedades Físico-Químicas dos Fluidos}

Os resultados das análises das propriedades físico-químicas dos fluídos a temperatura de $22^{\circ} \mathrm{C}$ foram: para a Água Destilada, Densidade $997 \mathrm{Kg} / \mathrm{m}^{3}$ e Viscosidade 0,0010 Pa.s; para o Óleo de Soja, Densidade $920 \mathrm{Kg} / \mathrm{m}^{3}$ e Viscosidade 0,0621 Pa.s. O resultado da tensão interfacial entre o óleo de soja/água destilada foi de $0,02013 \mathrm{~N} / \mathrm{m}$. O resultado do ângulo de contato medido entre o óleo de soja e o aço inoxidável submersos em água destilada foi de $72,7^{\circ}$.

\subsection{Etapa de Modelagem e Simulação}

Na Tabela 1 são apresentados os resultados do estudo preliminar de sensibilidade do ângulo de contato objetivando reproduzir o diâmetro médio de gota experimental. A definição desse ângulo exigiu um estudo cuidadoso, sendo que o valor que mais se adequou foi de $73^{\circ}$. Simulações posteriores, com melhor ajuste dos demais parâmetros, elevaram o percentual do diâmetro da gota simulada em relação ao experimental para cerca de $98 \%$.

Tabela 1 - Testes de ângulo de contato para a reprodução do diâmetro médio da gota experimental

\begin{tabular}{cc}
\hline Ângulos de contato testados $\left[^{\circ}\right]$ & $\begin{array}{c}\text { Percentual do Diâmetro Médio da gota simulada frente ao } \\
\text { experimental }\end{array}$ \\
\hline 65 & 88,70 \\
70 & 88,75 \\
71 & 88,75 \\
72 & 88,79 \\
73 & 89,15 \\
74 & 87,97 \\
75 & 88,61 \\
80 & 87,70 \\
85 & 86,80 \\
\hline
\end{tabular}

Conforme o procedimento apresentado no item 2.1., o ângulo de contato avaliado experimentalmente foi de $72,7^{\circ}$, o que demostra, apesar das limitações em outros aspectos, a robustez e potencialidade desse tipo de modelo para representação deste sistema experimental.

Os resultados da influência da variação da velocidade de injeção de óleo sobre a altura de gotejamento, diâmetro médio da gota formada e frequência de gotejamento estão apresentados na Tabela 2. Frente a esses resultados, pode ser observado que o aumento da vazão do óleo provoca um aumento da altura e frequência de gotejamento ao mesmo tempo que leva a uma pequena redução no diâmetro das gotas. Em geral, o modelo reproduziu adequadamente o tamanho das gotas. O maior desvio do modelo em relação ao experimento foi observado na tentativa de 
reprodução da altura de gotejamento para a menor velocidade de injeção; de fato, os maiores desvios foram encontrados para esse aspecto do fenômeno, o qual está certamente relacionado à frequência do gotejamento. Isto demonstra que o modelo carece de aprimoramentos principalmente na descrição das forças interfaciais, que aqui são consideradas mediante um único valor para a tensão interfacial. Em sistemas dinâmicos, a variação e composição dessas forças atuando ao nível da interface apresentam grande complexidade e são de difícil representação.

Tabela 2 - Resultados experimentais, simulados e desvio percentual para a altura de gotejamento, diâmetro médio da gota formada e tempo entre liberação de gotas

\begin{tabular}{|c|c|c|c|c|c|c|c|c|c|}
\hline \multirow[t]{2}{*}{ Dados } & \multicolumn{3}{|c|}{$\begin{array}{c}\text { Vel } 0,225 \mathrm{~m} / \mathrm{s} \\
\sigma=0,036[\mathrm{~N} / \mathrm{m}]\end{array}$} & \multicolumn{3}{|c|}{$\begin{array}{c}\text { Vel. } 0,333 \mathrm{~m} / \mathrm{s} \\
\sigma=0,038[\mathrm{~N} / \mathrm{m}]\end{array}$} & \multicolumn{3}{|c|}{$\begin{array}{c}\text { Vel } 0,446 \mathrm{~m} / \mathrm{s} \\
\sigma=0,036[\mathrm{~N} / \mathrm{m}]\end{array}$} \\
\hline & Exp & Sim & Desvio \% & Exp & Sim & Desvio \% & Exp & Sim & Desvio \% \\
\hline $\begin{array}{l}\text { Altura de } \\
\text { gotejamento, mm }\end{array}$ & 2,00 & 5,31 & $+165,5$ & 11,00 & 9,62 & $-12,55$ & 17,50 & 14,97 & $-14,46$ \\
\hline Diâmetro médio, mm & 5,53 & 5,52 & $-0,18$ & 5,24 & 5,26 & $+0,38$ & 4,65 & 4,59 & $-1,29$ \\
\hline Tempo entre Gotas, $s$ & 1,32 & 1,19 & $-9,85$ & 0,79 & 0,62 & $-21,52$ & 0,46 & 0,50 & $+8,70$ \\
\hline
\end{tabular}

Em seguida são apresentadas as imagens comparativas entre os experimentos e as simulações (Figuras 2 e 3), com imagens obtidas dos experimentos e através do software COMSOL que permite visualizar em cinza o isocontorno de fração de volume de 0,5; um corte central em escala de cores para ilustrar as diferentes velocidades apresentadas no domínio a cada instante e em preto os vetores proporcionais de velocidade.
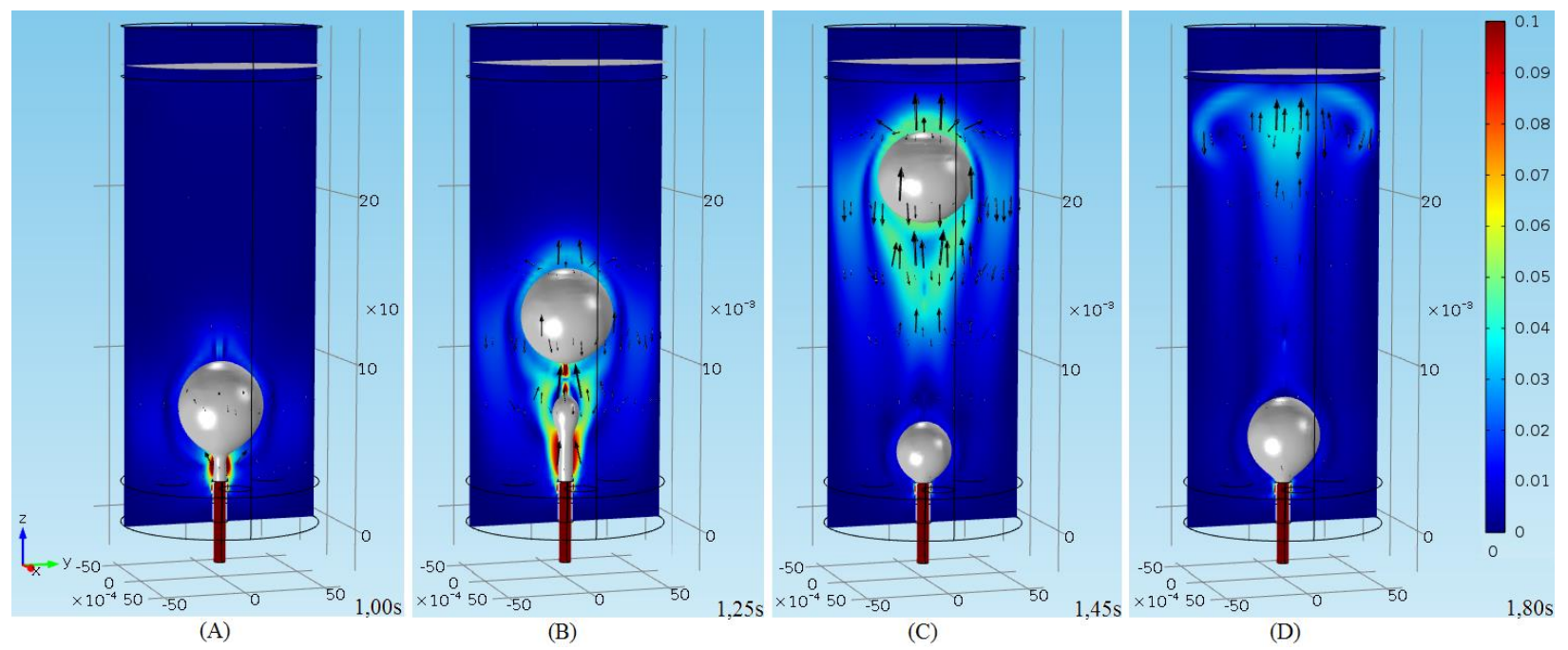

Figura 2 - (A), (B), (C) e (D) Imagens da simulação do escoamento de óleo/água para a velocidade de $0,225 \mathrm{~m} / \mathrm{s}$. Nos tempos de $1,0 \mathrm{~s} ; 1.25 \mathrm{~s}, 1,45 \mathrm{~s}$ e 1,8 s 
(A)

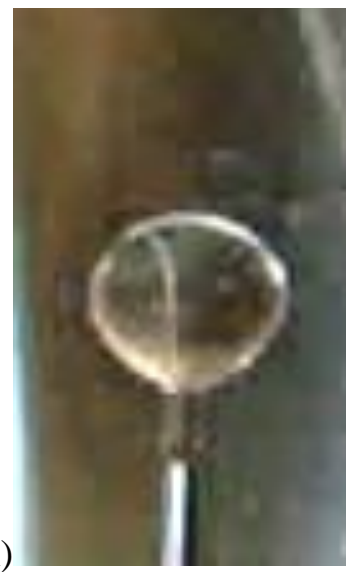

(B)

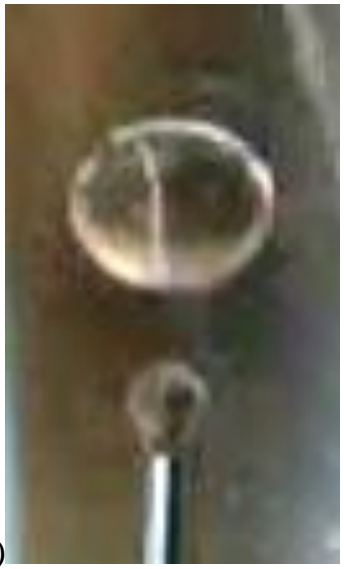

(C)

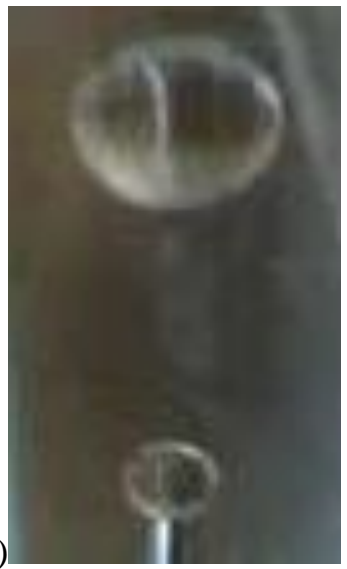

Figura 3 - (A), (B) e (C) Imagens experimentais para os tempos de 1,0 s; $1.25 \mathrm{~s}$ e 1,45 s

A Figura 2 A mostra a simulação óleo/água a $22^{\circ} \mathrm{C}$ injetada através do bocal cilíndrico a velocidade de $0,225 \mathrm{~m} / \mathrm{s}$ para o tempo de $1,00 \mathrm{~s}$, com a gota sendo formada e com o "pescoço" de óleo sendo delineado. Ainda na Figura 2 A, pode-se visualizar os vetores e a distribuição de velocidades no domínio. Os quadros $2 \mathrm{~A}$ e $3 \mathrm{~A}$ dessas figuras permitem tanto a análise quantitativa, (dados apresentados na Tabela 2), quanto qualitativa do momento de formação da gota. No tempo de 1,25 s (Figura 2 B), tem-se o instante do desprendimento de uma massa óleo e pode-se perceber a formação de uma zona de estagnação a certa distância da gota, pois os vetores ascendentes que acompanham o movimento da gota são anulados pelos vetores descendentes que promovem a recirculação do meio contínuo, guardando grande semelhança com a Figura 3 B que representa a realidade do sistema experimental. Na Figura $2 \mathrm{C}$ observa-se o movimento de ascensão da gota e na saída do bocal a formação de uma nova gota; os vetores de velocidade proporcionais são maiores na parte superior da massa em ascensão, sendo este o comportamento físico esperado para o sistema em estudo. Na Figura 2 D tem-se o momento após a coalescência da gota com a fase superior de óleo, onde fica evidente no corte de distribuição de velocidades a recirculação da fase contínua, apresentando simetria bilateral com orientação vertical, o que está de acordo com a disposição física esperada.

\section{CONCLUSÕES}

Os experimentos foram realizados para o sistema de injeção de óleo de soja em água destilada através do bocal cilíndrico, com três velocidades testadas. Na etapa experimental foram avaliadas as propriedades físico-químicas. $\mathrm{O}$ estudo de ângulo de contato apresentou diferença de $+0,41 \%$ entre o valor simulado e o experimental, demostrando a adequação do modelo para representar esse aspecto. De posse da caracterização experimental do sistema, foi possível realizar as simulações a partir de parâmetros realísticos, logrando-se obter uma boa representação do fenômeno tanto do ponto de vista qualitativo como quantitativo. Os resultados 
obtidos para o diâmetro médio da gota simulada apresentaram desvio máximo de 1,29 \%. Para o tempo entre a liberação das gotas, os desvios foram inferiores a 22,0 \%.

De outro lado, as simulações permitiram colocar em evidência os padrões de escoamento e distribuição de velocidades durante a formação, desenvolvimento, gotejamento e ascensão das gotas; aspectos esses de difícil quantificação experimental. Assim foi possível visualizar vórtices laterais que se formam quando ocorre a coalescência das gotas com a camada superior de óleo, bem como zonas de estagnação e recirculação junto às paredes do domínio.

Este conjunto de resultados atesta a pertinência da metodologia utilizada, estabelecendo este tipo de modelo como um excelente ponto de partida para a consideração de problemas de vazamento de óleo a partir de uma fonte submersa.

\section{REFERÊNCIAS}

KOBAYASHI, I., K. Uemura, et. al. CFD analysis of generation of soybean oil-in-water emulsion droplets using rectangular straight-through microchannels. Food Sci. and Technology Research, v. 13, p. 187-192, 2007.

LI Y., JAIN M. e NANDAKUMAR K. Numerical Study of Droplet Formation inside a Microfluidic Flow-Focusing Device. Excerpt from the Proceedings of the 2012 COMSOL Conference in Boston. 2012.

OLSSON, E.; KREISS, G. A Conservative Level Set method for two phase flow. J. of Comput. Physics, n. 210, pp. 225-246, 2005.

TIMGREN A., TRÄGÅRDH G. e TRÄGÅRDH C. CFD modelling of drop formation in a liquid-liquid system. $6^{\text {th }}$ International Conference on Multiphase Flow, ICMF 2007, Leipzig, Germany, July 9 - 13, 2007.

TORNBERG A., ENGQUIST B.. A finite element based level-set method for multiphase flow applications. Comput. Visual. Sci., 3, p. 93-101. 2000.

ZHI W., SHICHANG W., SCHROEDER V., SCHUBERT H., Influence of fluid flow on forces acting on droplet and emulsification results in membrane emulsification process. J. Chem. Ind. Eng. (China) v.50 (4), p. 505-513, (1999). 